\title{
YTHDF2 alleviates cardiac hypertrophy via regulating Myh7 mRNA decoy
}

\author{
Hongfei $\mathrm{Xu}^{1 \dagger}$, Zhen Wang ${ }^{1 \dagger}$, Miao Chen ${ }^{1 \dagger}$, Wenting Zhao ${ }^{2 \dagger}$, Tingting Tao ${ }^{1 \dagger}$, Liang Ma, Yiming $\mathrm{Ni}^{1 *}$ and \\ Weidong $\mathrm{Li}^{1 *}$
}

\begin{abstract}
Background: Pathological cardiac hypertrophy is a major contributor of heart failure (HF), which seriously threatens human's health world widely. Deregulation of m6A RNA methylation, and m6A methyltransferases and de-methyltransferases have been demonstrated to act essential roles in cardiac hypertrophy and HF. Here, we studied the potential roles and its underlying mechanisms of m6A Reader YTHDF proteins in HF. In this study, we constructed HF mouse model by transverse aortic constriction surgery. Primary cardiomyocytes were isolated and stimulated with isoproterenol (ISO) or phenylephrine (PHE) to induce myocardial hypertrophy.

Results: Through single-cell RNA-seq analysis, immunofluorescent staining, HE staining, Western blotting, and real time-PCR detections, we found that YTHDF2 mRNA and protein level, but not YTHDF1 or YTHDF3, was significantly increased during HF development. YTHDF2 overexpression could efficiently alleviate cardiac hypertrophy. Furthermore, through immunoprecipitation accompanied with mass spectrometry analysis, Gene Ontology (GO) analysis, and Kyoto Encyclopedia of Genes and Genomes (KEGG) pathway analysis, we found that ISO stimulation did not evidently affect YTHDF2-interacting proteins. However, ISO or PHE stimulation significantly increased YTHDF2 protein interacting with Myh7 (beta-myosin heavy chain) mRNA, an important cardiac hypertrophy marker, in an m6Adependent manner. Knockdown of Myh7 or deletion of the YTH domain of YTHDF2 reversed the protective effects of YTHDF2 on cardiac hypertrophy. Finally, we found that ISO or PHE stimulation promoted YTHDF2 protein expression through enhancing Ythdf2 mRNA stability in an m6A-dependent manner in cardiomyocytes.

Conclusions: Overall, our results indicate that the m6A Reader YTHDF2 suppresses cardiac hypertrophy via Myh7 mRNA decoy in an m6A-dependent manner. This study highlights the functional importance of YTHDF2-dependent cardiac m6A mRNA regulation during cardiac hypertrophy, and provides a novel mechanistic insight into the therapeutic mechanisms of YTHDF2.
\end{abstract}

Keywords: Heart failure, Cardiac hypertrophy, N6-methyladenosine (m6A) modification, YT521-B homology domain family (YTHDF) proteins, Myh7 (beta-myosin heavy chain)

*Correspondence: 1183020@zju.edu.cn; liweidong@zju.edu.cn

${ }^{\dagger}$ Hongfei Xu, Zhen Wang, Miao Chen, Wenting Zhao, Tingting Tao contributed equally to this work

${ }^{1}$ Department of Cardiovascular Surgery, School of Medicine, The First Affiliated Hospital of Zhejiang University, Number 79 Qingchun Road, Hangzhou, China

Full list of author information is available at the end of the article

\section{Introduction}

Heart failure (HF), characterized by reduced cardiac function and left ventricular dilatation, is one of the leading causes of death all over the world, and the 5year survival rate of patients with HF is only about $50 \%$ [1]. Pathological cardiac hypertrophy, generally triggered by pressure overload, is a critical risk factor of $\mathrm{HF}$ [2]. Cardiac hypertrophy is initially considered as an adaptive response to produce sufficient force to match

(c) The Author(s) 2021. This article is licensed under a Creative Commons Attribution 4.0 International License, which permits use, sharing, adaptation, distribution and reproduction in any medium or format, as long as you give appropriate credit to the original author(s) and the source, provide a link to the Creative Commons licence, and indicate if changes were made. The images or other third party material in this article are included in the article's Creative Commons licence, unless indicated otherwise in a credit line to the material. If material is not included in the article's Creative Commons licence and your intended use is not permitted by statutory regulation or exceeds the permitted use, you will need to obtain permission directly from the copyright holder. To view a copy of this licence, visit http://creativecommons.org/licenses/by/4.0/. The Creative Commons Public Domain Dedication waiver (http://creativecommons.org/publicdomain/zero/1.0/) applies to the data made available in this article, unless otherwise stated in a credit line to the data. 
an increase in wall tension or increased workload, but could eventually lead to HF [3]. So far, although great achievements have been made in the treatment for HF, there is still no effective drugs for completely preventing the eventual progression of the disease [4]. Thus, understanding the molecular events leading to HF is essential for supplying specific targets for novel drug research and development.

N6-methyladenosine (m6A) modification, the most abundant internal chemical modification in RNA, plays critical roles in regulating RNA processing, nuclear output, translation regulation, and RNA degradation [5, 6]. Generally, m6A modification in RNA is dynamically and reversibly regulated, and occurs within a highlyconserved consensus motif RRACH $(\mathrm{R}=\mathrm{G}$ or $\mathrm{A}, \mathrm{H}=\mathrm{A}$, $\mathrm{C}$ or $\mathrm{U}$ ) [7]. m6A modification is catalyzed by the methyltransferase complex, including methyltransferase-like 3 (METTL3), methyltransferase-like 14 (METTLl4), Wilms tumor 1-associated protein (WTAP), protein virilizer homolog (VIRMA), E3 ubiquitin-protein ligase Hakai (HAKAI), zinc finger $\mathrm{CCCH}$ domain-containing protein 13 (ZC3H13), RNA-binding protein 15 (RBM15), and methyltransferase-like 16 (METTL16) [8-10], and is removed by demethylases, such as ALKB homolog 5 (ALKBH5) and fat mass and obesity-associated protein (FTO) [11]. m6A modified RNA was recognized by "Reader" proteins, such as YT521-B homology domain family (YTHDF) proteins, to realize different biological functions [12-14]. Recently studies report that alteration of m6A RNA methylation contributes to HF progression [15], and enhanced m6A methylation leads to compensated cardiac hypertrophy [16]. Besides, cardiac-specific FTO knockout mice exhibit a more severe reduction in ejection fraction and a higher degree of dilatation upon transverse aortic constriction (TAC) surgery [15]. These findings indicate that m6A RNA methylation regulates cardiac homeostasis and hypertrophy. However, the molecular mechanisms of YTHDF proteins involved in HF progression are still unclear.

In this study, we explored the possible roles of YTHDF proteins during HF progression using human failing hearts samples and mice model of TAC. We found that YTHDF2 mRNA and protein level, but not YTHDF1 or YTHDF3, were significantly increased in HF tissues and cardiomyocytes with hypertrophic stimulation. YTHDF2 overexpression could efficiently alleviate cardiac hypertrophy. Furthermore, YTHDF2 protein interacts with Myh7 (beta-myosin heavy chain) mRNA, an important cardiac hypertrophy marker, to induce Myh7 mRNA decoy in an m6A-dependent manner, thereby inhibiting HF development. Our study highlights the functional importance of YTHDF2-dependent cardiac m6A mRNA regulation during heart failure, and provides a novel mechanistic insight into the therapeutic mechanisms of YTHDF2.

\section{Results \\ YTHDF2 protein expression increases in human heart failure samples}

Recent studies find that methylase Mettl3 and demethylase FTO function essential roles during HF progression through regulating m6A methylation level $[16,17]$. To explore the roles of YTHDF family proteins during HF, we analyzed recently published single-cell RNA-seq data of TAC-model mouse heart tissue (GSE120064) [17], and found 5 major cellular clusters, including cardiomyocytes $(\mathrm{CM})$, endothelial cells $(\mathrm{EC})$, fibroblasts $(\mathrm{FB})$, macrophages (MP), and smooth muscle cells (SMC) distributed in the HF tissue (Fig. 1A). Besides, more cardiomyocytes distributed in the left ventricle (LV) than in the left atrial (LA) (Fig. 1A). Furthermore, we found YTHDF $1 / 2 / 3$ proteins are distributed in all of the 5 major cellular clusters (Fig. 1A). Subsequently, we analyzed the expression levels of YTHDF1/2/3 in the clinical normal and HF tissues, and found that YTHDF2 mRNA levels (Fig. 1B) and protein levels (Fig. 1C, D) in the HF tissues were significantly higher than those in the normal heart tissues. As expected, the protein expression levels of Atrial Natriuretic Peptide (ANP) were significantly increased in the HF tissues, which is consistent with previous study [18] (Fig. 1C). Moreover, the wheat germ agglutinin (WGA) staining of myocardial tissue showed that myocardial cells in the HF tissues were larger than those the normal heart tissues, and immunofluorescent staining further confirmed that YTHDF2 protein expressions were notably increased in the HF tissues (Fig. 1E). Collectively, these results suggest that YTHDF2 protein increases in human HF samples.

\section{YTHDF2 protein expression increases in the cell and animal model of cardiac hypertrophy}

Subsequently, we further investigated the expression levels of YTHDF $1 / 2 / 3$ in the primary cardiomyocytes treated with ISO or PHE to induce myocardial hypertrophy in vitro, and in the mice with cardiac hypertrophy induced by TAC in vivo. As shown in Fig. 2A-C, YTHDF2 mRNA and protein expression level was significantly increased in either ISO or PHE treated cardiomyocytes, compared to control. Whereas, ISO or PHE stimulation did not obviously affect YTHDF1 and YTHDF3 mRNA and protein expression. Furthermore, consistent with the in vitro results, YTHDF2 mRNA and protein expression level was also significantly increased in the mice with cardiac hypertrophy induced by TAC (Fig. 2D-F). Interestingly, YTHDF1 protein, but not mRNA expression level 


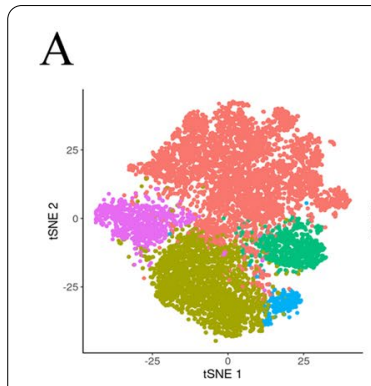

ident $\cdot \mathrm{CM} \cdot \mathrm{EC} \cdot \mathrm{FB} \cdot \mathrm{MP} \cdot \mathrm{SMC}$

B

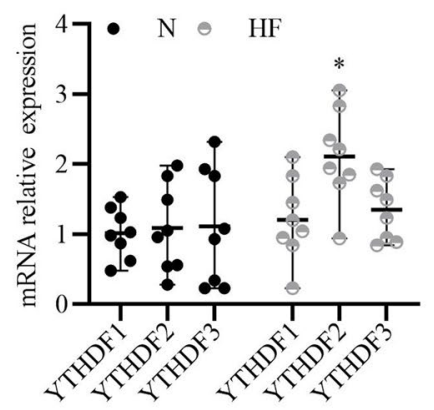

$\mathrm{C}$

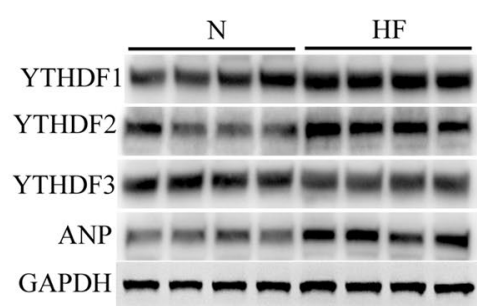

$\mathrm{D}$

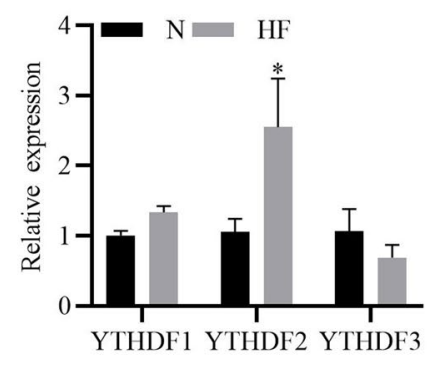

$\mathrm{E}$
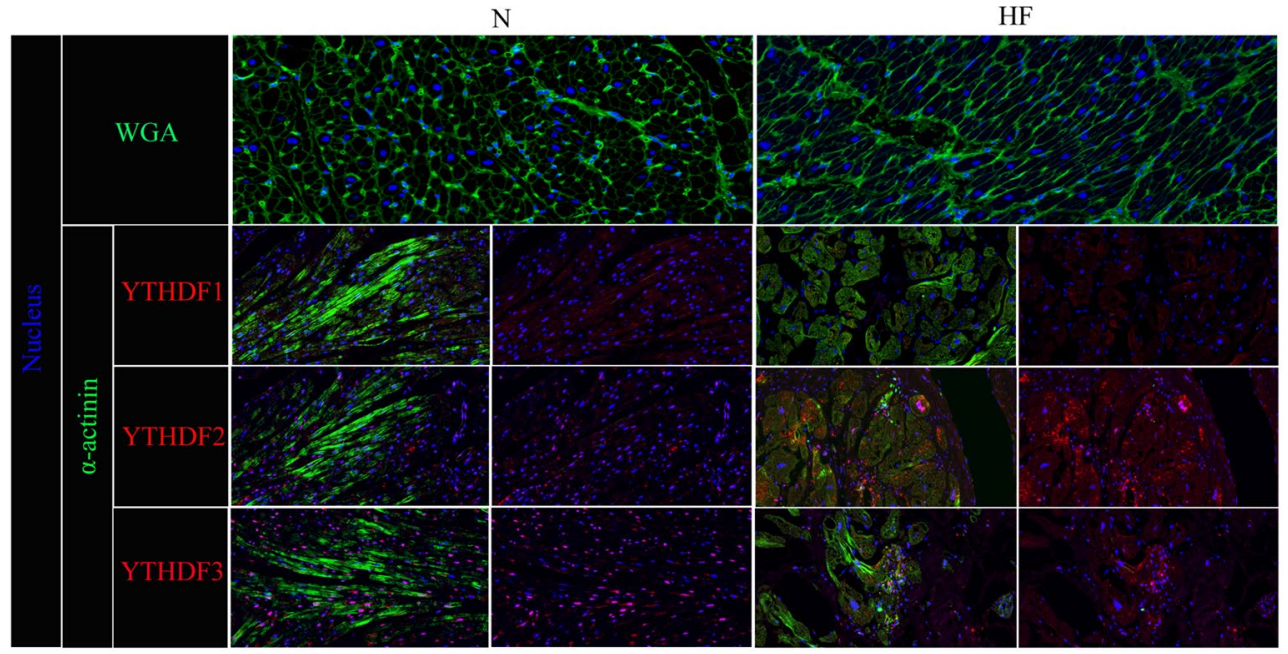

Fig. 1 YTHDF2 protein expression increases in human heart failure samples. A 2D visualization of single-cell clusters of TAC-model mouse heart tissues by tSNE. (CM cardiomyocytes; EC endothelial cells, FB fibroblasts, MP macrophages, SMC smooth muscle cells); $2 D$ visualization of single-cell clusters present in the left ventricle (LV) and the left atrial (LA); 2D visualization of YTHDF1/2/3 gene expressions in the single-cell clusters present in the left ventricle. B RT-PCR analysis of YTHDF1/2/3 mRNA expressions in the clinical HF $(n=8)$ tissues and the normal $(N)$ heart tissues $(n=8)$. C Western blotting analysis of YTHDF1/2/3 protein expressions in the clinical HF $(n=4)$ tissues and the normal $(N)$ heart tissues $(n=4)$. ANP was used as a positive control, and GAPDH was used as a loading control. D Densitometry quantification of protein expressions. E WGA staining (Green) was used to measure cardiomyocyte size of the clinical HF tissues and the normal (N) heart tissues; Immunofluorescence analysis of YTHDF1/2/3 (Red) expressions in cardiomyocytes (stained with anti-a-actinin antibodies, Green). Nucleus was stained with DAPI (Blue). ${ }^{*} P<0.05$

also significantly upregulated in the mice with cardiac hypertrophy induced by TAC. Moreover, immunofluorescent staining further confirmed that YTHDF2 protein expressions were notably increased in the hypertrophic mice hearts (Fig. 2G). Collectively, these results suggest that YTHDF2 was highly expressed in the hypertrophic mice hearts and ISO or PHE-induced cardiomyocytes. 


\section{YTHDF2 plays a protective role on cardiac hypertrophy} in vivo

To further explore the specific role of YTHDF2 during cardiac hypertrophy development, gain/loss-offunction experiments were performed. The mice were injected with AAV9 viral particles carrying YTHDF2 (AAV-YTHDF2) through tail intravenous injection, or control AAV for 3 weeks, and the mice were later subjected to TAC to induce cardiac hypertrophy. As shown in Fig. 3A-D, YTHDF2 overexpressing mice showed a smaller heart size (Fig. 3A), lower HW/BW (Fig. 3B), and fewer enlarged cardiomyocytes (Fig. 3C, D), compared with the control mice after 4 weeks of TAC surgery. Consistently, YTHDF2 overexpressing mice also showed less fibrosis detected by the Masson staining (Fig. 3E), and lower LVEDd measured by echocardiographic evaluation (Fig. 3F). Moreover, as expected, YTHDF2-knockdown mice exhibited a larger heart size (Fig. 3G), higher $\mathrm{HW} / \mathrm{BW}$ (Fig. 3H), more enlarged cardiomyocytes (Fig. 3I, J), more fibrosis (Fig. 3K), and higher LVEDd (Fig. 3L). Collectively, these results indicate that YTHDF2 overexpression alleviates cardiac hypertrophy in vivo.

\section{Functional analysis of YTHDF2-interacting proteins in cardiomyocytes stimulated with ISO}

To further investigate the molecular mechanism of YTHDF2 alleviating cardiac hypertrophy, YTHDF2interacting proteins in the cardiomyocytes treated with DMSO (as control) or ISO were immunoprecipitated and then identified by mass spectrometry. As shown in Fig. 4A, B, GO and KEGG annotation enrichment analyses showed that ISO stimulation did not significantly affect the molecular functions or associated with diseases of YTHDF2 interacting proteins. Besides, proteinprotein interaction (PPI) network analysis showed that ISO stimulation did not significantly affect the interactions between YTHDF2 and the proteins identified by mass spectrometry (Fig. 4C). Moreover, transcriptional regulation analysis showed that ISO stimulation did not obviously affect the transcription of the genes coding proteins potentially interacted with YTHDF2 (Fig. 4D). Among the identified proteins by mass spectrometry,
MYH7 (beta-myosin heavy chain) has been reported as an important cardiac hypertrophy marker [19]. Thus, we firstly hypothesized whether ISO stimulation affects the interaction between YTHDF2 and MYH7. Co-IP analysis showed that YTHDF2 indeed interacted with MYH7 in cardiomyocytes. But, ISO stimulation did not affect the interaction of YTHDF2 and MYH7 (Fig. 4E), suggesting YTHDF2 may be not affect cardiac hypertrophy by interacting with MYH7. In addition, RNA immunoprecipitation (RIP) analysis showed that ISO stimulation did not affect YTHDF2 interacted with SRF (serum response factor) mRNA or BRCA1 (Breast carcinoma 1) mRNA (Fig. 4F).

\section{YTHDF2 alleviating myocardial hypertrophy depends on interacting with Myh7 mRNA}

Next, we further explored whether the RNA binding ability of YTHDF2 is involved in YTHDF2 alleviating cardiac hypertrophy. Plasmid expressing YTH domaindelated YTHDF2 (YTH-del), which is responsible for YTHDF2's RNA binding ability [20], was synthesized. As shown in Fig. $5 \mathrm{~A}-\mathrm{C}$, consistent with the results of Fig. 3C, overexpression of YTHDF2 evidently suppressed ISO or PHE-induced myocardial hypertrophy, whereas abolishment of YTHDF2's RNA binding ability partially reversed the effect of overexpression of YTHDF2, suggesting that YTHDF2-mediated RNA metabolism is involved in the progression of cardiac hypertrophy. Furthermore, we detected whether YTHDF2 interacts with the mRNAs of cardiac hypertrophy makers, including ANP (atrial Natriuretic Peptide), BNP (brain natriuretic peptide), and MYH7 by RIP. The results showed that ISO or PHE stimulation evidently increased the interaction of YTHDF2 and Myh7 mRNA, but not Anp or Bnp mRNA (Fig. 5D). Furthermore, we found that knockdown of MYH7 significantly inhibited ISO or PHE-induced myocardial hypertrophy, and overexpression of YTHDF2 did not further alleviate myocardial hypertrophy (Fig. 5E-G). In addtion, RIP analysis showed that YTHDF2 directly binds to Myh7 mRNA, but deletion of the YTH

\footnotetext{
(See figure on next page.)

Fig. 2 YTHDF2 protein expression increases in the heart tissues of mice with cardiac hypertrophy. A RT-PCR analysis of YTHDF1/2/3 mRNA expressions in the primary cardiomyocytes stimulated with isoproterenol (ISO, $10 \mu \mathrm{mol} / \mathrm{I})$, phenylephrine (PHE, $50 \mu \mathrm{mol} / \mathrm{I})$, or DMSO (as control) for $24 \mathrm{~h}$. B Western blotting analysis of YTHDF1/2/3 protein expressions in the primary cardiomyocytes stimulated with ISO (+, $10 \mu \mathrm{mol} / \mathrm{l} ;++$, $20 \mu \mathrm{mol} / \mathrm{l}), \mathrm{PHE}(+, 50 \mu \mathrm{mol} / \mathrm{l} ;++, 80 \mu \mathrm{mol} / \mathrm{l})$, or DMSO (as control) for $24 \mathrm{~h}$. C Densitometry quantification of protein expressions. D RT-PCR analysis of YTHDF1/2/3 mRNA expressions in the heart tissues of mice after 4 weeks of TAC surgery $(n=6)$ or Sham surgery $(n=6)$. $\mathbf{E}$ Western blotting analysis of YTHDF1/2/3 protein expressions in the heart tissues of Sham $(n=6)$ or TAC $(n=6)$ mice. $\mathbf{F}$ Densitometry quantification of protein expressions. G WGA staining (Green) was used to measure cardiomyocyte size in the heart tissues of Sham or TAC mice; Immunofluorescence analysis of YTHDF1/2/3 (Red) expressions in cardiomyocytes (stained with anti-a-actinin antibodies, Green). Nucleus was stained with DAPI (Blue). ${ }^{*} P<0.05$
} 
A

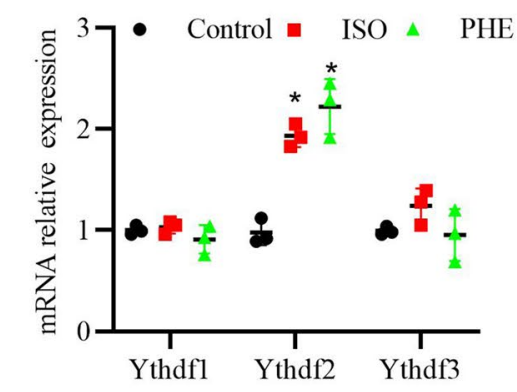

D

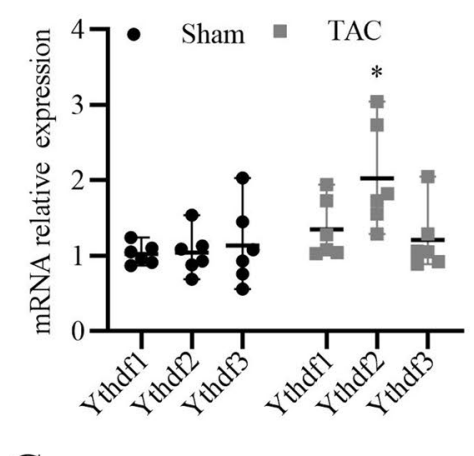

B

C

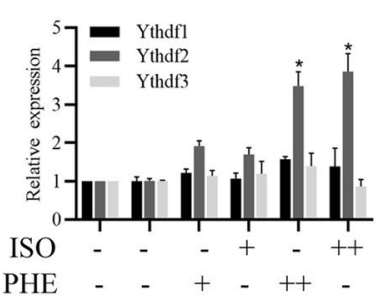

E

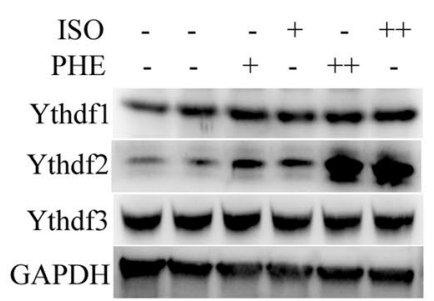

F

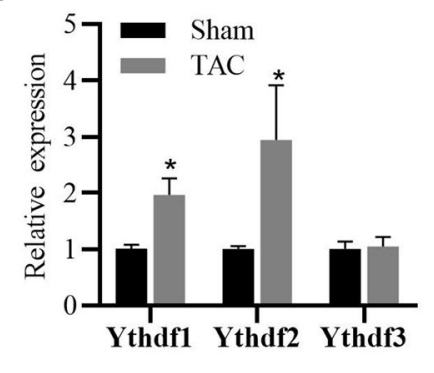

G

Sham

TAC

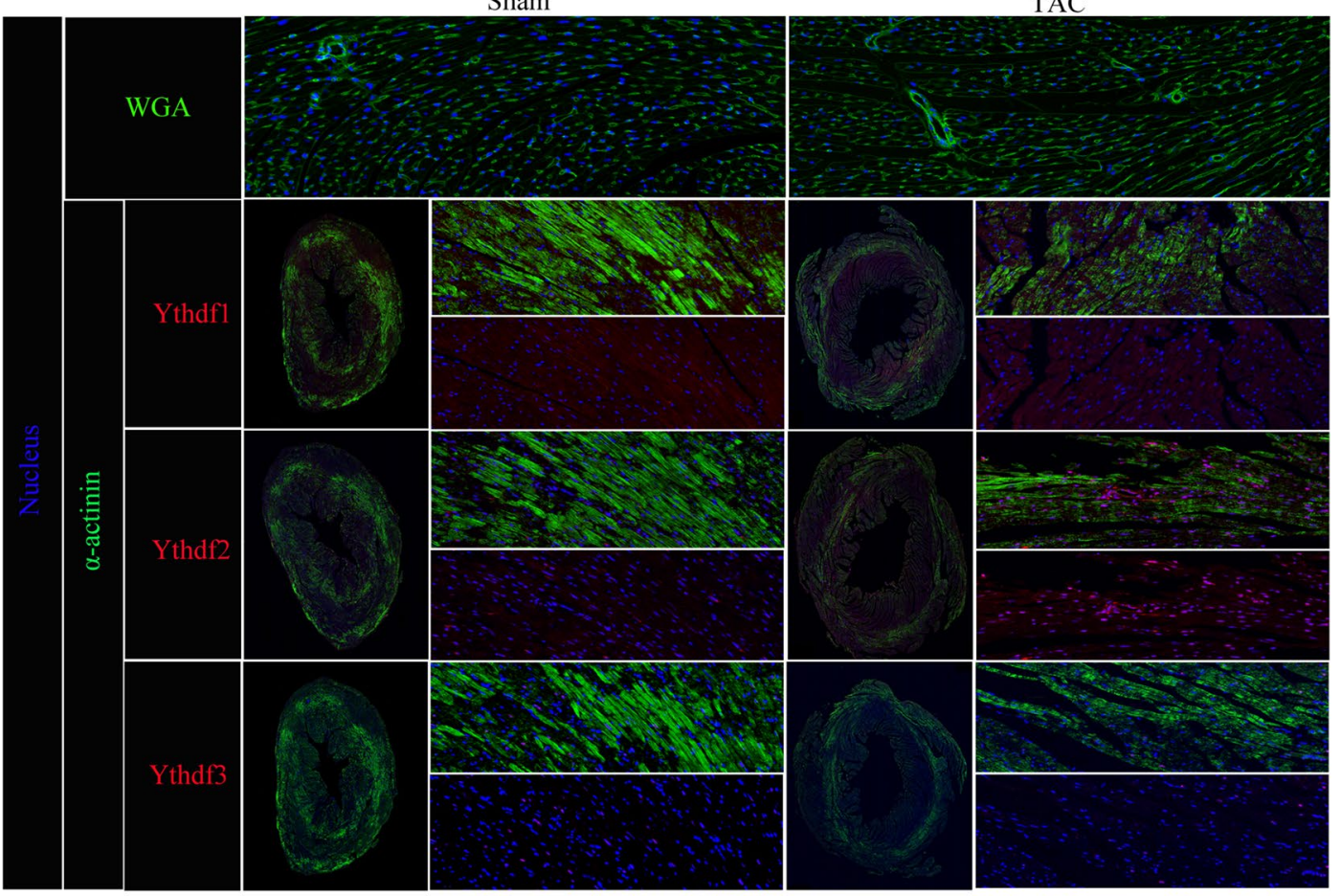

Fig. 2 (See legend on previous page.) 
domain of YTHDF2 cancels this binding (Fig. 5H). Collectively, these results indicate that YTHDF2 interacting with Myh7 mRNA via YTH domain to alleviate cardiac hypertrophy.

\section{YTHDF2 recognizes the $\mathrm{m} 6 \mathrm{~A}$ site on Myh7 mRNA to promote its degradation}

Given that YTHDF2 interacting with Myh7 mRNA in cardiomyocytes (Fig. 5D), we further explored whether YTHDF2 affected MYH7 expression. As shown in Fig. 6A, B, knockdown of YTHDF2 did not affect MYH7 protein stability (Fig. 6A) analyzed by protein stability assay, whereas knockdown of YTHDF2 significantly increased Myh7 mRNA stability (Fig. 6B). Furthermore, YTHDF2 knockdown did not affect the distribution of Myh7 mRNA in the polysome (F13-F14) or subpolysome fractions (F3-F4) (Fig. 6C), indicating that YTHDF2 did not affect Myh7 mRNA translation. Considering that m6A modification plays important roles in regulating mRNA decoy [21], we further whether m6A modification occurs on Myh7 mRNA. RIP analysis with anti-m6A antibodies showed that m6A modification occurs in the CDS (coding sequence)- 6 region of Myh7 mRNA (Fig. 6D), and two potential m6A modification-motif were identified in the region (Fig. 6E). Subsequently, pGL3-luciferase reporter plasmid coding the wide type (WT) or mutant (Mut) sequences of CDS-6 region of Myh7 mRNA was transfected into Hela cell lines. Then, RIP analysis showed that WT, but not Mut plasmid was specifically immunoprecipitated with anti-m6A antibodies (Fig. 6F). Moreover, YTH domaindelated YTHDF2 (YTH-del) significantly canceled the binding of WT Myh7 CDS-6 but not Mut (Fig. 6G). In addition, RNA pulldown analysis with biotinylated-Myh7 mRNA CDS-6 (WT) or mutant Myh7 mRNA CDS-6 (Mut) probe showed that WT Myh7 mRNA CDS-6 specifically interacted with YTHDF2 protein (Fig. 6H). Overall, these results indicate that YTHDF2 recognizes the m6A site on Myh7 mRNA to promote its degradation.
ISO or PHE stimulation promotes YTHDF2 protein expression through enhancing Ythdf 2 mRNA stability in cardiomyocytes

To further explore the underlying mechanism of YTHDF2 upregulation during HF progression, we firstly examined the interaction between RNA polymerase II, which is mainly responsible for transcriptional initiation of gene [22], and Ythdf2 gene promoter regions [range from 0 to $-2000 \mathrm{bp}$ from transcription start site, including region-1 (0 to $-1000 \mathrm{bp})$ and region-2 (- $1001 \mathrm{bp}$ to $2000 \mathrm{bp}$ )]. The result of CHIP with antibodies against RNA polymerase II showed that ISO or PHE stimulation did not significantly affect Ythdf2 gene transcription in cardiomyocytes, but evidently promoted Bnp gene transcription (Fig. 7A). Recently, Histone H3 lysine 4 (H3K4) trimethylation (H3K4me3), generally correlates with transcriptional activation of genes [23], has been reported to play essential roles in cardiac homeostasis [24]. We further examined the effects of ISO or PHE on the H3K4me3 modification of Ythdf2 gene, and CHIP analysis showed that ISO or PHE stimulation did not significantly affect H3K4me3 modification of Ythdf2 gene (Fig. 7B), but significantly promoted H3K4me3 modification on Bnp gene. Besides, ISO or PHE stimulation also did not affect the distribution of Ythdf 2 mRNA in the polysome fraction (F13-F14) or subpolysome fractions (F3-F4) [25, 26] (Fig. 7C). Furthermore, we detected the effects of YTHDF1/3 proteins on Ythdf2 expression, and found that knockdown of YTHDF1 or YTHDF3 did not affect Ythdf2 mRNA or protein expressions (Fig. 7D-F). Unexpected, RIP analysis showed that ISO or PHE stimulation significantly decreased YTHDF2 protein interacting with Ythdf2 mRNA (Fig. 7G), suggesting YTHDF2 may have a self-regulation. Moreover, ISO or PHE stimulation evidently suppressed Ythdf2 mRNA degradation in cardiomyocytes (Fig. $7 \mathrm{H}$ ), indicating that YTHDF2 protein could regulate Ythdf 2 mRNA stability under ISO or PHE stimulation. Taken together, these results indicate that ISO or PHE stimulation promotes YTHDF2

\footnotetext{
(See figure on next page.)

Fig. 3 YTHDF2 plays a protective role on cardiac hypertrophy in vivo. A HE staining for the assessment of gross cardiac enlargement of mice injected with AAV-YTHDF2 or control AAV through tail vein for 3 weeks, then subjected to TAC for 4 weeks. B Statistical analysis of the heart weight $(\mathrm{HW})$ /body weight (BW) ratios of mice subjected to TAC surgery for 4 weeks ( $n=6$ mice per group; ${ }^{*} p<0.05$ versus sham group). $\mathbf{C}$ WGA staining (Green) was used to measure cardiomyocyte size in the heart tissues of TAC mice. D Statistical analysis of the cross section areas of the indicated group. E Representative results of the Masson staining to assess fibrosis of the mouse heart tissues. $\mathbf{F}$ Detection of left ventricular end-diastolic diameter (LVEDd) in mice, $\mathrm{n}=6$. G HE staining for the assessment of gross cardiac enlargement of mice injected with AAV-si-YTHDF2 or control AAV through tail vein for 3 weeks, then subjected to TAC for 4 weeks. $\mathbf{H}$ Statistical analysis of the heart weight (HW)/body weight (BW) ratios of mice subjected to TAC surgery for 4 weeks ( $n=6$ mice per group; ${ }^{*} p<0.05$ versus sham group). I WGA staining (Green) was used to measure cardiomyocyte size in the heart tissues of TAC mice. J Statistical analysis of the cross section areas of the insdicated group. $\mathbf{K}$ Representative results of the Masson staining to assess fibrosis of the mouse heart tissues. L Detection of left ventricular end-diastolic diameter (LVEDd) in mice, $\mathrm{n}=6$. ${ }^{*} P<0.05$
} 


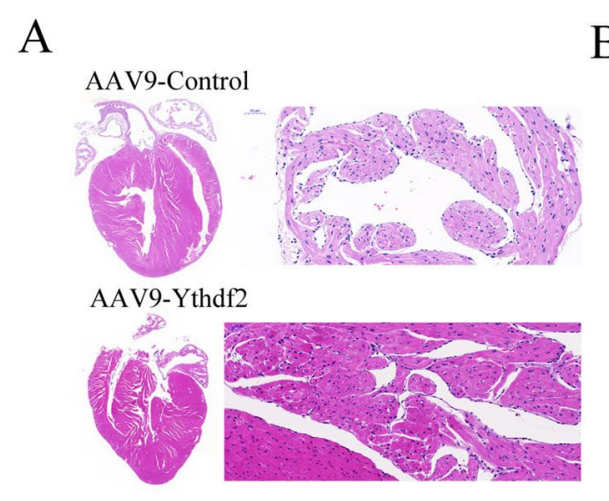

D

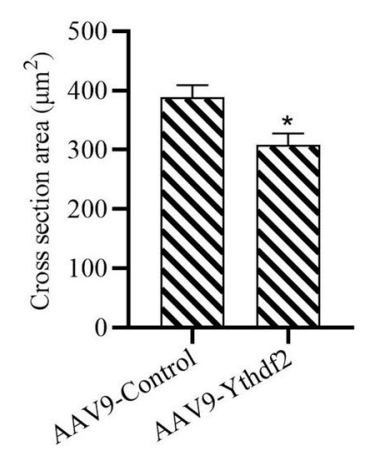

G

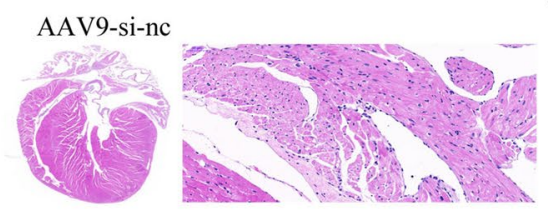

AAV9-si-Ythdf2

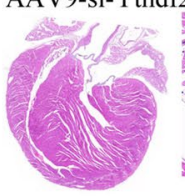

J

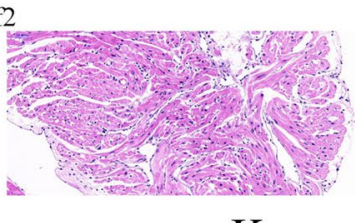

K
B

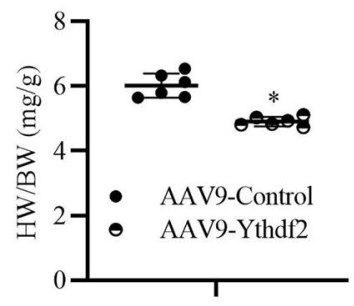

AAV9-Control
C

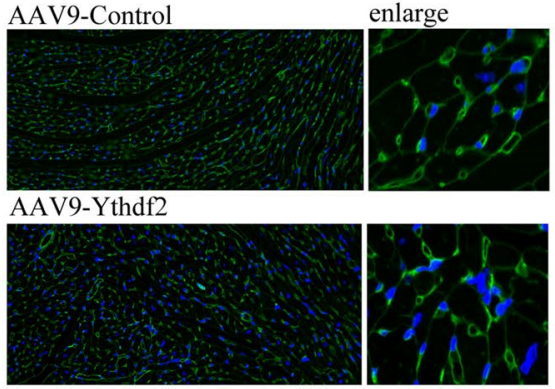

F

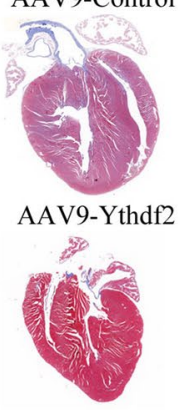

$\mathrm{H}$
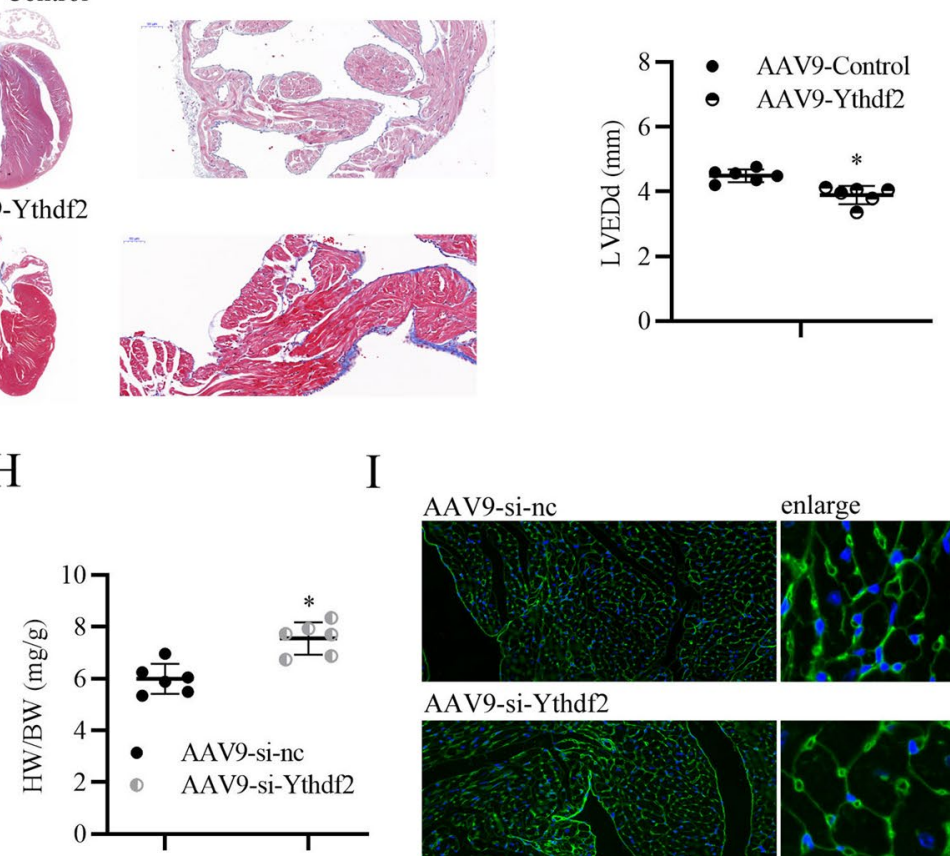

I

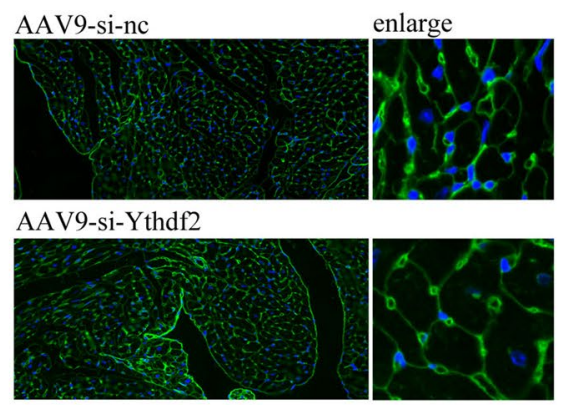

L
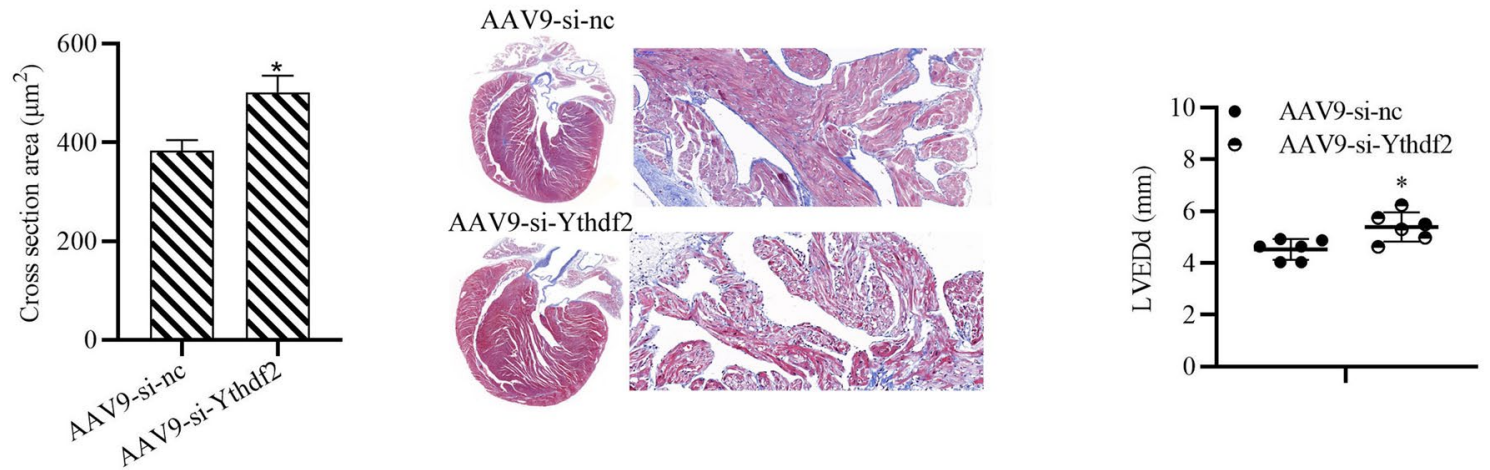

Fig. 3 (See legend on previous page.) 


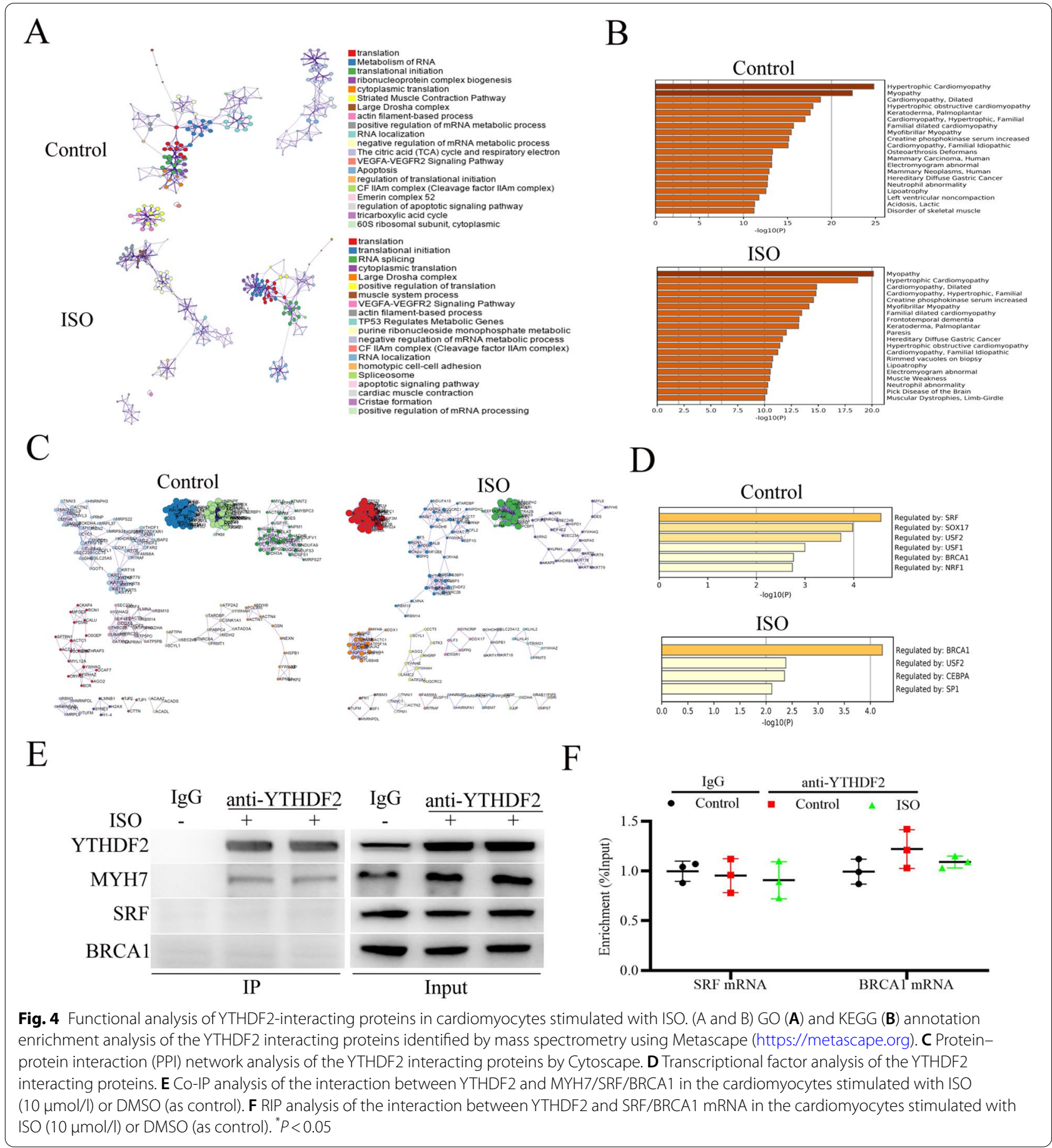

self-regulation and protein upregulation through enhancing Ythdf2 mRNA stability in cardiomyocytes.

\section{Discussion}

HF, which leads to most of cardiovascular hospitalizations and death all over the world, still lacks therapeutic targets and effective drugs nowadays [27].
Remodeling of the m6A landscape plays an important role in the development of cardiomyocyte hypertrophy and HF [28]. m6A content has been reported to be increased in human heart failure samples, and increased expression of the m6A RNA methylase METTL3 was sufficient to promote cardiomyocyte hypertrophy both in vitro and in vivo [28]. 


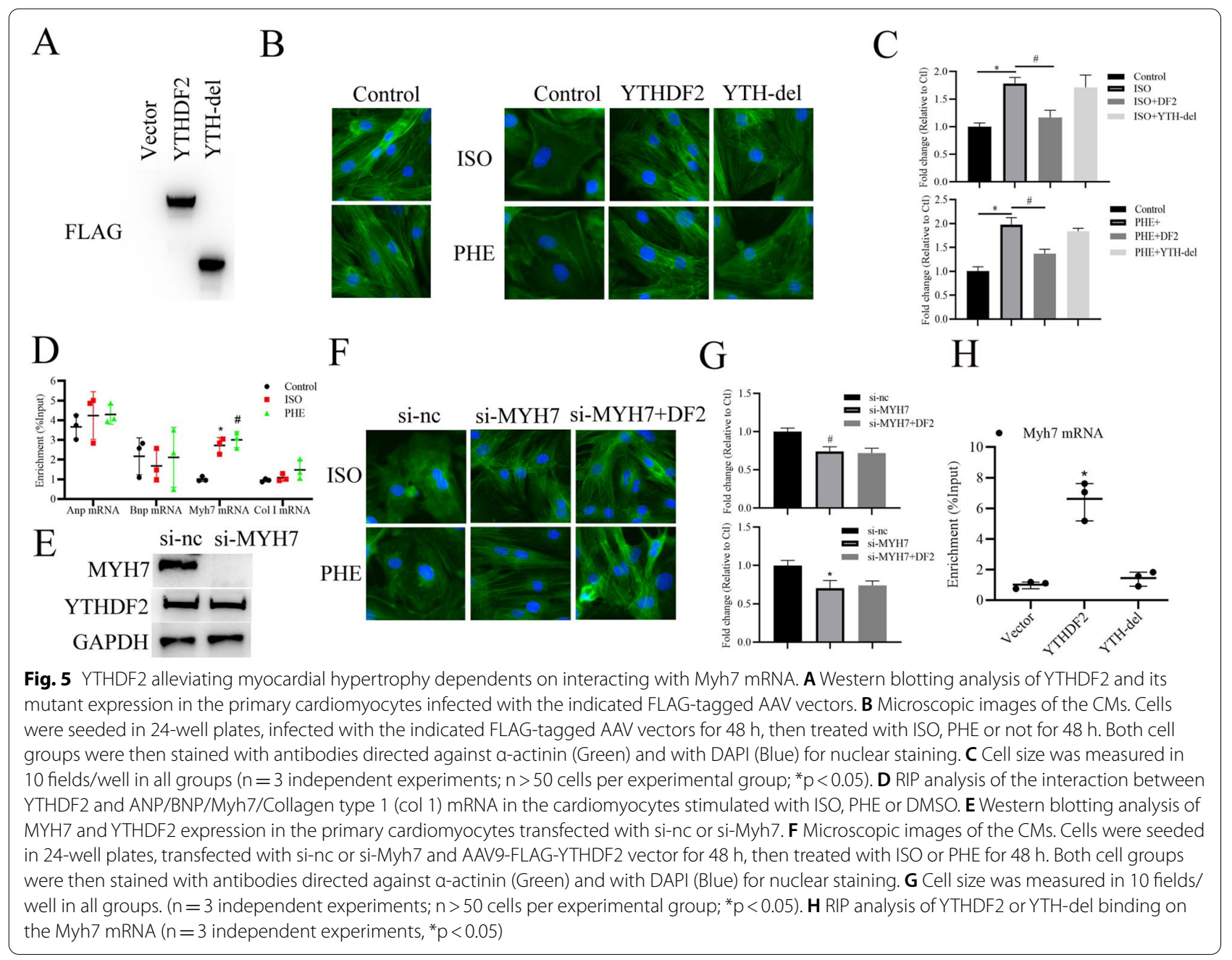

Consistently, upregulation of m6A RNA demethylase FTO expression in failing mouse hearts improves cardiac contractile function [29]. In this study, we for the first time explored the roles of the m6A "Reader" YTHDF family during HF development, and found that YTHDF2, but not YTHDF1 or YTHDF3, increases in human HF samples, mice HF samples, and cardiomyocytes with hypertrophic stimulation. Furthermore, we found that YTHDF2 suppresses cardiac hypertrophy via Myh7 mRNA decoy in an m6A-dependent manner. Our study highlights the functional importance of m6A "Reader" proteins-dependent cardiac m6A mRNA regulation during $\mathrm{HF}$, and provides a novel mechanistic insight into the therapeutic mechanisms of YTHDF2.

YTHDF family proteins, including YTHDF1, YTHDF2 and YTHDF3, positively or negatively regulate RNA metabolism through recognizing $\mathrm{m} 6 \mathrm{~A}$ modification via YTH domain. YTHDF1 is reported to promote the translation of m6A mRNA by enhancing the ribosome assembly of m6A mRNA and interacting with the initiation factor [12]. YTHDF2 mainly regulates the stability of target mRNA through transforming the m6A RNA from the translation state to the degradation state [30]. YTHDF3 can promote the translation and degradation of mRNA [31]. In this study, we find that YTHDF2 specifically interacts with Myh7 mRNA, an important cardiac hypertrophy marker, to induce Myh7 mRNA decoy in an m6a-dependent manner in cardiomyocytes, and hypertrophic stimulation further enhances the interaction of YTHDF2 and Myh7 mRNA. To date, more than 400 hypertrophic cardiomyopathy-associated mutations have been described in Myh7 gene [32], and patients with likely pathogenic or pathogenic variation in Myh7 gene have a higher rate of incident atrial fibrillation independent of clinical and echocardiographic factors [33]. Whether m6A modification is associated with Myh7 gene mutation needs more attention in the following studies. Besides, we also find that YTHDF2 interacts with MYH7 protein in cardiomyocytes, whereas 


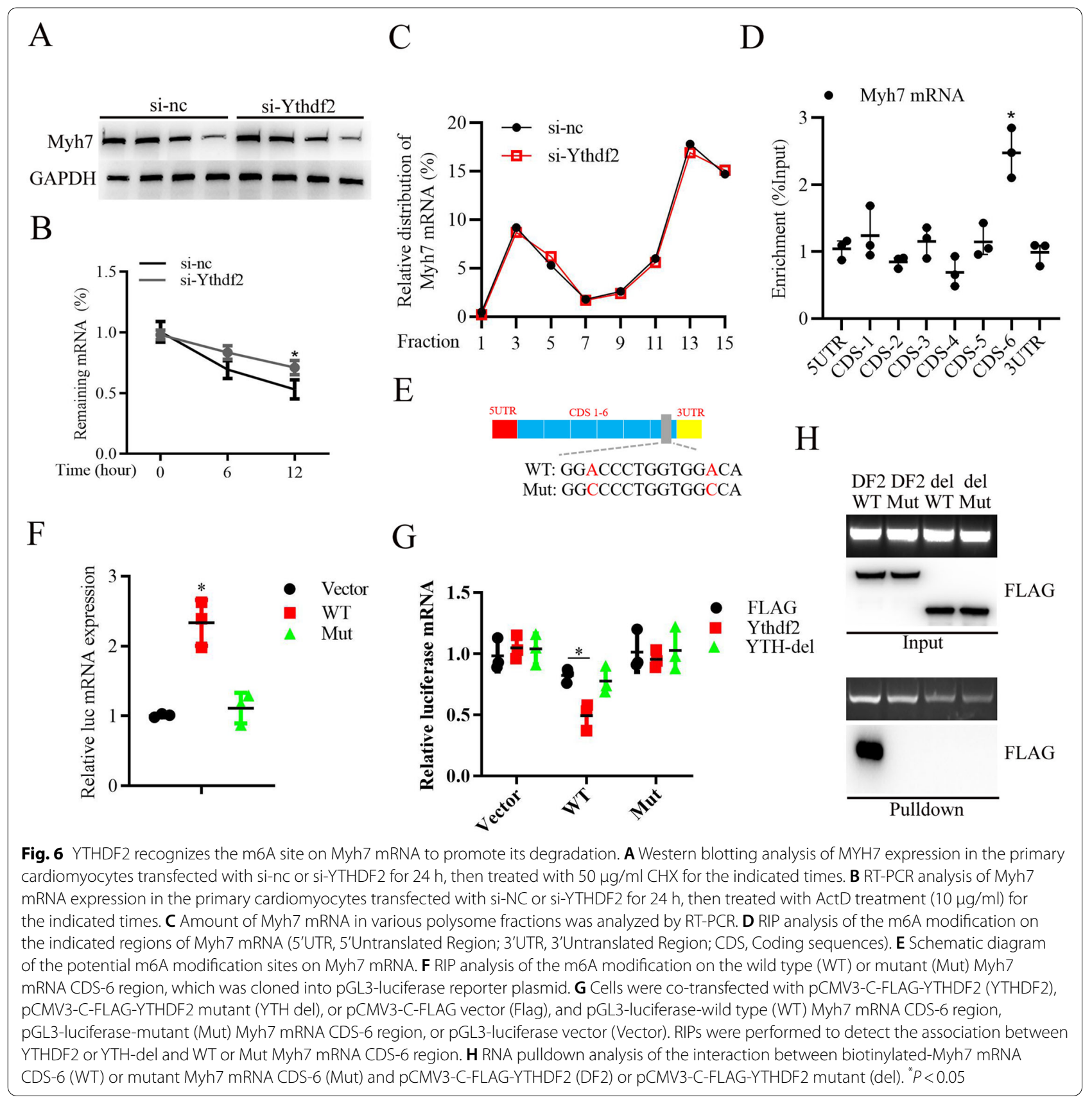

hypertrophic stimulation does not affect the interaction of YTHDF2 and MYH7 protein. Whether YTHDF2 interacting with $\mathrm{MYH} 7$ protein influences myocardial active contraction, such as isometric force levels, shortening velocity, and calcium sensitivity of force generation, needs to be further studied. In addition, even if no significant differences of YTHDF1/3 expression between the normal heart tissues and HF tissues, the detailed roles of YTHDF1/3 during HF development still require deeper investigation.
As an m6A reader protein, YTHDF2 participates in various biological processes, including migration, invasion, metastasis, proliferation, apoptosis, cell cycle, cell viability, cell adhesion, differentiation and inflammation, in many diseases [34-36]. Through immunoprecipitation accompanied with mass spectrometry analysis, we find that the proteins-interacted with YTHDF2 in cardiomyocytes are mainly related to hypertrophic cardiomyopathy, myopathy and dilated cardiomyopathy, suggesting YTHDF2 may function essential roles on 


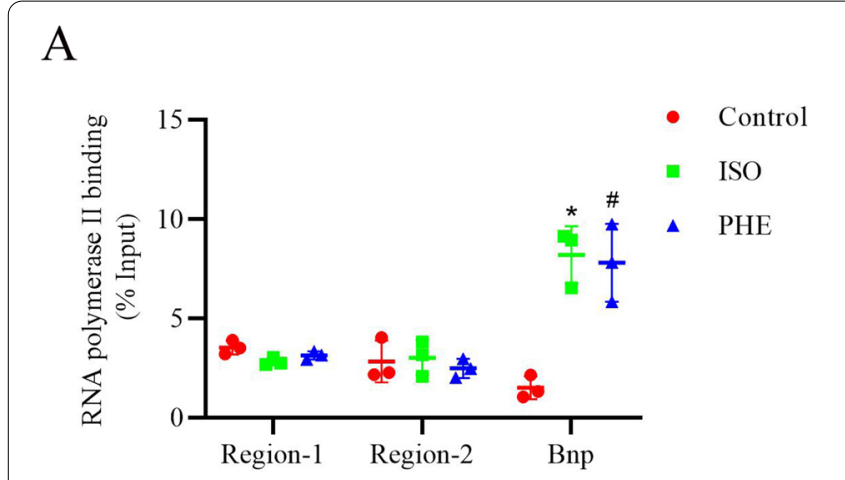

B

C

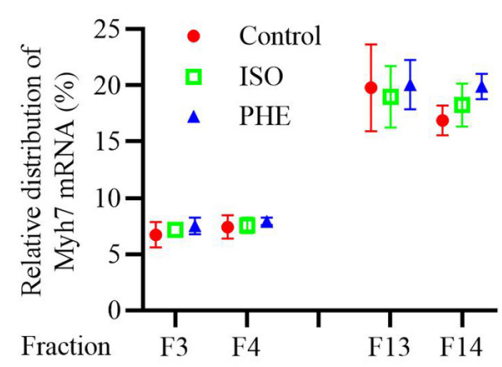

F

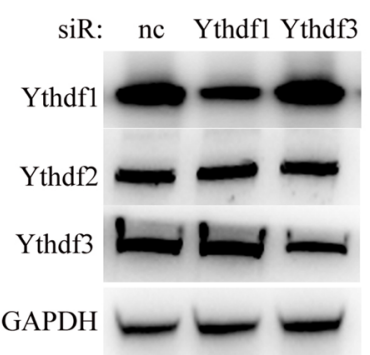

D

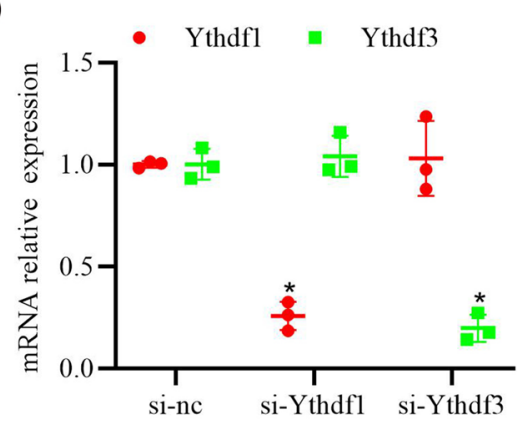

G

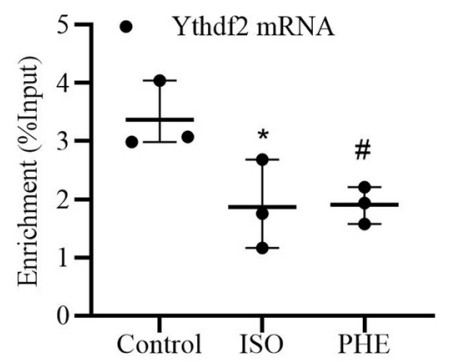

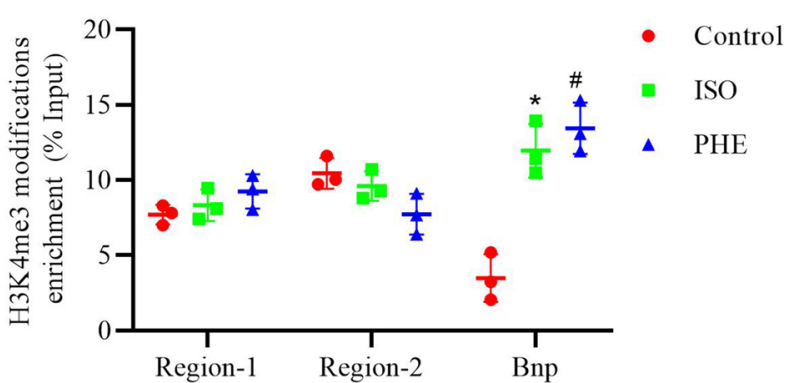

E

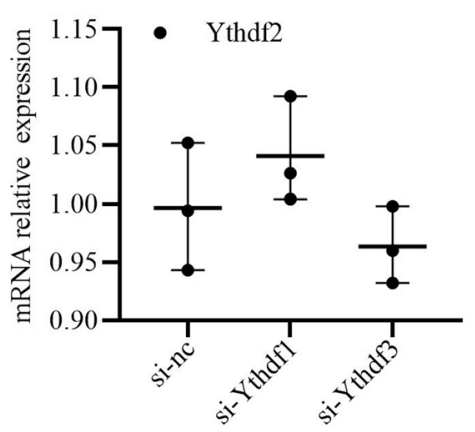

$\mathrm{H}$

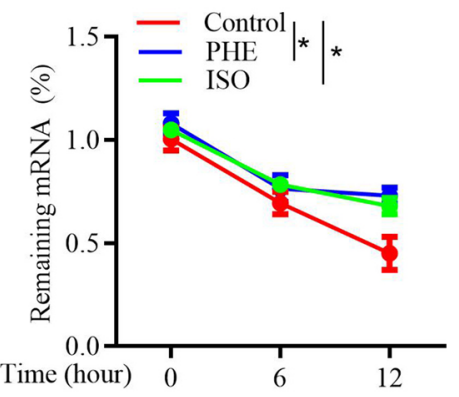

Fig. 7 ISO or PHE stimulation promotes YTHDF2 protein expression through enhancing Ythdf2 mRNA stability in cardiomyocytes. A CHIP analysis of the interaction between RNA polymerase II and YTHDF2 gene promoter region 1/2 or BNP gene promoter in cardiomyocytes stimulated with ISO, PHE or DMSO (as control). B CHIP analysis of H3K4me3 modification on the YTHDF2 gene promoter region 1/2 or BNP gene promoter in cardiomyocytes stimulated with ISO, PHE or DMSO (as control). CRT-PCR analysis of the amount of YTHDF2 mRNA in various polysome fractions in cardiomyocytes stimulated with ISO, PHE or DMSO (as control). D RT-PCR analysis of YTHDF1/3 mRNA expressions in cardiomyocytes transfected with si-nc, si-YTHDF1, or si-YTHDF3. E RT-PCR analysis of YTHDF2 mRNA expressions in cardiomyocytes transfected with si-nc, si-YTHDF1, or si-YTHDF3. F Western blotting analysis of YTHDF1/2/3 protein expressions in cardiomyocytes transfected with si-nc, si-YTHDF1, or si-YTHDF3. G RIP analysis of the interaction between YTHDF2 protein and YTHDF2 mRNA in cardiomyocytes stimulated with ISO, PHE or DMSO (as control). H RT-PCR analysis of YTHDF2 mRNA expression in the primary cardiomyocytes treated with ActD treatment $(10 \mu \mathrm{g} / \mathrm{ml})$ for the indicated times. ${ }^{*} P<0.05$

cardiac homeostasis. Among the identified proteins, MYH6 ( $\alpha$-myosin heavy chain) and MYH9 play important roles in heart development, and are associated with HF [37, 38]. In addition, Protein arginine methyltransferase 5 (PRMT5), a protein arginine methyltransferase that catalyzes the symmetrical dimethylation of arginine residues within target proteins, is an important regulator of myocardial hypertrophic signaling [39]. Besides regulating mRNA decoy, whether the protective effect of YTHDF2 on cardiac hypertrophy and HF also related to these proteins requires further investigation in the following studies.

In this study, we find that YTHDF2 suppresses cardiac hypertrophy through regulating Myh7 mRNA decoy in vitro using the primary mice cardiomyocytes stimulated with ISO or PHE, and in vivo using TAC mouse 
model. It is reported that the Myh isoforms are differentially expressed in the ventricular myocardium of rodents and humans [40-45]. The Myh composition of the ventricular myocardium of rodents is major $\alpha-\mathrm{Myh}$ (Myh6) [40-42], whereas that of humans is major $\beta$-Myh (Myh7) [43-45]. Thus, hiPSC (human induced pluripotent derived stem cell)-differentiated cardiomyocytes should be used to further uncover the regulatory mechanism of YTHDF2 on human cardiac hypertrophy. In addition, although the differential Myh composition of the ventricular myocardium of rodents and humans, the protein level alteration of Myh (down-regulation of $\alpha$-Myh and up-regulation of $\beta$-Myh) was consistent in both human HF and experimental animal HF models [46]. In rats, $\alpha$-Myh protein could decrease from $93-97 \%$ to $22-71 \%$, while $\beta-$ Myh protein could increase from $3-6 \%$ to $29-78 \%$ during heart failure [46]. For the humans, $\alpha$-Myh mRNA was expressed at considerable levels (30\% of total MyHC mRNA) in the non-failing human left ventricles and was substantially decreased (reduced to 2\%) in end-stage failing human ventricles [47]. The Myh isoform switching is a major contributing factor to the decline in cross-bridge kinetics observed in experimental rodent HF models [49]. Whether YTHDF2 is also involved in regulating the Myh isoform switching during human HF progression needs to be investigated in the following studies.

In conclusion, out study explores the role of YTHDF family proteins during HF development, and indicates YTHDF2 suppresses cardiac hypertrophy and HF via Myh7 mRNA decoy in an m6A-dependent manner. This study highlights the functional importance of YTHDF2dependent cardiac m6A mRNA regulation during heart failure, and sheds light on potential new targets for hypertrophy and HF therapy.

\section{Methods and materials}

Single-cell RNA sequencing (scRNA-seq) analysis

Single cell RNA sequence data from heart tissues of TAC-model mice was obtained from the Gene Expression Omnibus (GEO) database (GSE120064) [17]. Cells expressing $<200$ or $>7000$ genes in normal heart samples, and $<200$ or $>5000$ genes in heart failure samples were filtered out for exclusion of noncell or cell aggregates. The data were log-normalized and highly variable genes were selected using the FindVariableFeatures function and downstream procedures were performed using the ScaleData and runPCA function. Then, FindClusters function was performed to cluster cells and uniform manifold approximation and projection (UMAP) with $\mathrm{R}$ package Seurat was used to visualize clusters, as described in the vignettes (https://satijalab.org/seurat/ vignettes.html).

\section{Patient samples}

Human left ventricular samples were collected and used in this study, including control samples (nonfailing, nontransplantable hearts, $\mathrm{n}=8$ ), and heart failure samples (end-stage dilated cardiomyopathy, $\mathrm{n}=8$ ). All heart failure patients were diagnosed with dilated cardiomyopathy with $\mathrm{EF}<25 \%$ (systolic heart failure). All heart failure patients were diagnosed with heart failure (Function Capacity IV, Objective Assessment D, based on New York Heart Association functional classifcation) at least 3 months before heart transplantation. Written informed consent was obtained from patients. Samples were collected in accordance with human research protocol approved by the Research Ethics Committee of the First Affiliated Hospital, College of Medicine, Zhejiang University.

\section{RNA extraction and real-time qPCR analysis}

The total RNA of heart tissues and isolated mice primary cardiomyocytes was extracted with TriZol reagent (Invitrogen, USA), and then reverse-transcribed into cDNA using PrimeScript RT Master Mix (Takara, Japan). SYBR Premix Ex TaqII (Takara, Japan) kit was used for qRTPCR detection with ABI PRISM 7500 Detection System (ABI, USA). Values were normalized by GAPDH. The primers are listed in Additional file 1: Table S1.

\section{Western blotting}

Proteins were extracted from heart tissues or isolated mice primary cardiomyocytes with RIPA Lysis Buffer (Beyotime, China), and protein concentration was determined using the BCA protein assay kit (Beyotime, China). Protein samples were separated on a 10\% SDS-PAGE gel and then transferred to PVDF membranes (Millipore, USA). The membranes were blocked with $5 \%$ non-fat milk for $1 \mathrm{~h}$ at room temperature and then incubated overnight at $4{ }^{\circ} \mathrm{C}$ with the following primary antibodies: YTHDF1 (ab220162, Abcam, USA), YTHDF2 (ab246514, Abcam, USA), YTHDF3 (ab220161, Abcam, USA), ANP (ab262703, Abcam, USA), flag (ab205606, Abcam, USA), and GAPDH (ab8245, Abcam, USA), followed by incubation with secondary antibodies for $1 \mathrm{~h}$. The protein blots were visualized using the ECL kit (Pierce, USA).

\section{Transverse aortic constriction (TAC) mouse model}

All animal experimental procedures were approved by the Animal Care Ethics Committee of the first affiliated hospital Zhejiang university school of medicine, and the experiments were performed in compliance with the "Guide for the Care and Use of Laboratory Animals" from the US National Institute of Health. Eight-week-old male C57BL/6 J mice were purchased from Model Animal Research Center of Nanjing University, and housed 
in the Laboratory Animals Center of the first affiliated hospital Zhejiang university school of medicine, with controlled temperature and humidity. HF was induced in mice by transverse aortic constriction (TAC), as previously described [50]. Briefly, mice were anesthetized with $0.3 \%$ sodium pentobarbital $\left(75 \mathrm{mg} \cdot \mathrm{kg}^{-1}\right)$ intraperitoneally, and the aortic arch was tied with a 6-0 nylon suture between the brachiocephalic and left common artery with a homemade L-shaped 26G cushion needle. After ligation, the needle was quickly removed, and the skin was closed. The sham operation was identical, except that the thread was not ligated. Moreover, mice were injected with rAAV9 $\left(4 \times 10^{11}\right.$ vector genomes $(\mathrm{vg}) /$ mouse) carrying an empty vector, YTHDF2 or YTH-del via the tail vein.

\section{Cell isolation and culture}

Primary cardiomyocytes were isolated from adult mice (male, 8 weeks old) or neonatal mice (1-2 days). Briefly, after wash, hearts were cut up in saline with HEPESbuffer. Then, the tissues were decentralized and incubated in HEPES-buffered saline solution with $0.14 \mathrm{mg} / \mathrm{ml}$ collagenase and $1.2 \mathrm{mg} / \mathrm{ml}$ pancreatin at $37^{\circ} \mathrm{C}$. The cells collected by centrifugation were re-suspended in Dulbecco's modified Eagle medium/F-12 with $0.1 \mathrm{mM}$ ascorbate, $5 \%$ heat-inactivated fetal bovine serum (FBS), $100 \mathrm{U} / \mathrm{ml}$ penicillin, insulin-transferring-sodium selenite media supplement, $0.1 \mathrm{mM}$ bromodeoxyuridine and $100 \mu \mathrm{g} /$ $\mathrm{ml}$ streptomycin. After pre-plated at $37^{\circ} \mathrm{C}$ for $1 \mathrm{~h}$, the cells were plated in dishes coated by $10 \mu \mathrm{g} / \mathrm{ml}$ laminin. For inducing hypertrophy, cells were treated with treated with isoproterenol (ISO, $10 \mu \mathrm{mol} / \mathrm{l}$, Sigma, USA) or phenylephrine (PHE, $50 \mu \mathrm{mol} / \mathrm{l}$, Sigma, USA) for $24 \mathrm{~h}$.

\section{Echocardiographic evaluation}

4 weeks after TAC surgery, the cardiac function of mice by echocardiography using a 30-MHz high-frequency scanhead (VisualSonics Vevo770, VisualSonics, Canada). End-systole and end-diastole were defined as the phases in which the smallest and largest areas of the left ventricular (LV), respectively, were obtained. LV end-diastolic diameter (LVEDd) were measured from the LV M-mode at the mid-papillary muscle level. After echocardiographic evaluation, mice were euthanized by cervical dislocation 4 weeks post-operatively. Mice hearts were dissected and weighed or measured to compare the heart weight $(\mathrm{HW})$ /body weight $(\mathrm{BW})$ ratios.

\section{Histological study}

The heart tissue sections were paraffin embedded and cut into $5-\mu \mathrm{M}$ serial sections and then were stained with a hematoxylin and eosin (HE) staining kit (Byotime, China) to assess myocardial pathological changes, and a Masson staining kit (SbjBio, China) was used to evaluate cardiac fibrosis, respectively, according to the manufacture's instruction.

\section{Immunofluorescence}

The heart tissue sections were blocked with goat serum at room temperature for $30 \mathrm{~min}$, and then stained with fluorescein conjugated wheat germ agglutinin staining (Alexa Fluor-488, Invitrogen, CA) was used to evaluate cardiomyocyte size. Myocyte nucleus was stained using 4', 6-diamidino-2-phenylindole (DAPI, Sigma, USA). For each group, approximately 50-100 randomly chosen cardiomyocytes were analyzed by using Image J software to measure the cross-sectional cardiomyocyte area. To evaluate the expressions of YTHDF $1 / 2 / 3$ proteins in heart tissue, heart tissue sections were blocked, incubated with primary antibodies against $\alpha$-actinin (Sigma, USA) and YTHDF1/2/3 (Abcam, USA) at $4{ }^{\circ} \mathrm{C}$ overnight, and treated with fluorescence-conjugated secondary antibody for $1 \mathrm{~h}$ at $37^{\circ} \mathrm{C}$. The nuclei were counterstained with DAPI. After washing, the sections were imaged by a Zeiss Confocal Microscope Imaging System (Carl Zeiss, Germany).

\section{Co-immunoprecipitation (Co-IP) assay and mass spectrometry analysis}

Primary cardiomyocytes were treated with ISO or DMSO (as control) for $24 \mathrm{~h}$. Then, the cells were lysed with Cell lysis buffer for Western and IP (Beyotime, China), and centrifuged to collect the supernatant. One tenth of the supernatant was retained for the input immunoblot, while the rest (300 $\mu \mathrm{g}$ proteins) was incubated with antiYTHDF2 or rabbit IgG at $4{ }^{\circ} \mathrm{C}$ overnight, followed by further incubation with $10 \mu \mathrm{l}$ protein A/G-agarose beads (Cell Signaling Technology, USA) for another $4 \mathrm{~h}$. The bound proteins were subjected to washing three times for a total of $30 \mathrm{~min}$ and then eluted by boiling for $5 \mathrm{~min}$ in the loading buffer. Immunocomplexes were subjected into SDS-PAGE electrophoresis and the gel was then stained with the Fast Silver Stain Kit (Beyotime, Shanghai, China). Then, the gel was analyzed independently by reverse-phase liquid chromatography coupled with tandem mass spectrometry using ACQUITYTM UPLCQTOF analysis platform at Beijing Protein Innovation (Beijing, China). For detecting the interaction between YTHDF2 and MYH7/SRF/BRCA1, the immunoprecipitated proteins were resolved by SDS-PAGE and detected by Western blotting with anti-bodies against $\mathrm{MYH7}$ (22,280-1-AP, ProteinTech, China), SRF (ab252868, Abcam, USA), or BRCA1 (ab238983, Abcam, USA). 


\section{Bioinformatic analysis}

Metascape (http://metascape.org/gp/index.html\#/main/ step1) was applied to analyze the Gene ontology (GO) functional, disease association and transcription factors annotation of the proteins immunoprecipitated with YTHDF2 in primary cardiomyocytes treated with ISO or DMSO (as control).

\section{RNA-binding protein immunoprecipitation assay (RIP assay)}

Magna RIP Kit (Millipore, USA) was used to perform the RIP assay. In brief, Primary cardiomyocytes were treated with ISO or DMSO (as control) for $24 \mathrm{~h}$, and then lysed with RIP lysis buffer. The supernatant was incubated with antibodies against YTHDF2 antibody (Abcam, USA) overnight at $4{ }^{\circ} \mathrm{C}$. Then, $50 \mu \mathrm{l} \mathrm{A} / \mathrm{G}$ magnetic beads were added to the supernatant and incubated for $6 \mathrm{~h}$. After immobilizing the magnetic bead bound complexes with a magnetic separator (Millipore USA), supernatants were used to extract RNA with PCA (phenol: chloroform: isoamyl alcohol) reagent at a ratio of 125:24:1 (Aladdin, USA). The results were quantified by RT-PCR.

\section{siRNAs transfection}

siRNA against mouse Ythdf2 mRNA, and siRNA against mouse Myh7 mRNA were synthesized by GnenPharma (Shanghai, China). Plasmids or siRNAs were transiently transfected into cardiomyocytes using jetprime transfection reagents (Polyplus, France) according to the manufacturer's instructions.

\section{Protein and mRNA stability assays}

Primary cardiomyocytes were transfected with si-NC or si-Ythdf2 for $24 \mathrm{~h}$, then treated with $50 \mu \mathrm{g} / \mathrm{ml}$ cycloheximide (CHX; Sigma Aldrich) to block protein synthesis, or $5 \mathrm{mg} / \mathrm{ml}$ of Actinomycin D (ActD; Sigma Aldrich) to block RNA transcription. After culturing at the various time points, cells were collected for Western blotting, or subjected to RNA extraction. RT-PCR were used to analyze the levels of MYH7 mRNA.

\section{Polysome profiling}

Primary cardiomyocytes were transfected with si-NC or si-Ythdf2 for $24 \mathrm{~h}$, then treated with $100 \mu \mathrm{g} / \mathrm{ml}$ of cycloheximide for $15 \mathrm{~min}$. Then cells are lysed by polysome buffer $[200 \mathrm{mmol} / \mathrm{L} \mathrm{KCl}, 15 \mathrm{mmol} / \mathrm{L} \mathrm{MgCl} 2,1 \%$ Triton X100, $100 \mu \mathrm{g} / \mathrm{mL}$ cycloheximide, $20 \mathrm{mmol} / \mathrm{L}$ heparin, and $100 \mathrm{U} / \mathrm{mL}$ RNase Inhibitor (Takara), 1X cocktail] for $15 \mathrm{~min}$ on ice, lysates were centrifuged $(14,000 \mathrm{rpm}$ for $15 \mathrm{~min}$ ), and the supernatant was layered onto a 5 to $50 \%$ sucrose gradient. Gradients were then centrifuged at $38,000 \mathrm{rpm}$ for $130 \mathrm{~min}$ at $4{ }^{\circ} \mathrm{C}$ and polysome-bound fractions were collected using an ISCO Density Gradient
Fractionation System (ISCO, Lincoln, NE) with continuous monitoring based on A260nm wavelength. The RNA in each fraction was extracted using Trizol reagent (Invitrogen) and analyzed by RT-PCR .

\section{Luciferase assay}

pGL3-basic luciferase reporter vector containing the wild-type and mutant Myh7 mRNA CDS-6 sequences (NCBI Reference Sequence: NM_001361607.1) was synthesized by GnenPharma (Shanghai, China), respectively. $293 \mathrm{~T}$ cells were transfected with $750 \mathrm{ng}$ of pGL3-basic luciferase reporter vector (Vector), wild-type pGL3-Myh7 mRNA CDS-6 (WT) or mutant pGL3-Myh7 mRNA CDS-6 (Mut) in combination with pCMV3-CFLAG vector, pCMV3-C-FLAG-YTHDF2 plasmid, or pCMV3-C-FLAG-YTHDF2 YTH-del plasmid using lipo2000 transfection reagent (Life Technologies, USA). At $24 \mathrm{~h}$ after transfection, we lysed the cells and measured the luciferase activity by luciferase reporter assay system (Beyotime Biotechnology, Shanghai, China). All of the experiments were performed in triplicate.

\section{Biotin-coupled probe RNA pull down assay}

Biotinylated-Myh7 mRNA CDS-6 (WT) or mutant Myh7 mRNA CDS-6 (Mut) probe was synthesized by RiboBio (Guangzhou, China). Plasmids or siRNAs were transiently transfected into cardiomyocytes using jetprime DNA transfection reagents (Polyplus, France) according to the manufacturer's instructions. Cardiomyocytes were transfected with pCMV3-C-FLAG vector, pCMV3-CFLAG-YTHDF2 plasmid, or pCMV3-C-FLAG-YTHDF2 YTH-del plasmid using jetprime DNA transfection reagents (Polyplus, France). After transfection for $48 \mathrm{~h}$, the cells were collected and lysed. The lysate was then incubated with biotin-labeled probe. Next, the biotin-coupled RNA complex was pulled down using streptavidin-coated magnetic beads adsorption. The enriched YTHDF2 was analyzed by western blot analysis.

\section{Chromatin immunoprecipitation (ChIP)}

Chip assay was performed with the simple ChIP Enzymatic Chromatin IP Kit (Magnetic Beads) (Cell Signaling Technologies, USA), according to the manufacturer's instructions. Briefly, $5 \times 10^{6}$ cardiomyocytes were treated with ISO, PHE, or DMSO (as control) for $24 \mathrm{~h}$. Then, cells were cross-linked with $37 \%$ formaldehyde (final concentration of $1 \%$ ) and incubated for $10 \mathrm{~min}$ at room temperature. The chromatin fraction was digested with micrococcal nuclease and sonicated to obtain the desired fragment length of 150-900 bp. The protein-DNA complexes were immunoprecipitated using anti-RNA polymerase II (abcam, ab252854), or H3K4me3 (abcam, ab213224) antibodies. Then, the immunoprecipitated 
DNA was amplified by RT-PCR. The primers used are listed in Additional file 1: Table S1. The signals obtained from each immunoprecipitation reaction were expressed as a percent of the total input chromatin with the following equation:

$$
\begin{aligned}
\text { Enrichment percentage }= & 2 \% \times 2 \mathrm{CT} \text { input samples } \\
& - \text { CTIP samples }
\end{aligned}
$$

\section{Statistical analysis}

All statistical analysis was performed by GraphPad Prism 7.0. Data were expressed as mean \pm standard deviation (SD). Differences between two groups were determined by using unpaired Student's t-test. Furthermore, differences among multiple groups were determined by using ANOVA. Statistical significance was considered when $\mathrm{P}<0.05$.

\section{Supplementary Information}

The online version contains supplementary material available at https://doi. org/10.1186/s13578-021-00649-7.

Additional file 1: Table S1. Primers used in this study.

\section{Acknowledgements}

We thank Jie Zan for suggestions for the study.

\section{Authors' contributions}

$X H F, N Y M$ and L WD conceived and designed the experiments. X HF, W Z, C $M, T T T$ and ZWT performed the experiments and analyzed the data. X HF and LWD wrote the manuscript. All authors read and approved the manuscript.

\section{Funding}

This work was supported by Natural Science Foundation of Zhejiang Province, Zhejiang, China, Grant /Award Number: LQ19H070002 and LGF18H020006.

\section{Availability of data and materials}

All data generated and/or analyzed during this study are included in this published article.

\section{Declarations}

\section{Ethics approval and consent to participate}

The patients who donated the samples have signed informed consent, and the project was approved by the Ethics Committee of The First Affiliated Hospital of Zhejiang University Hospital.

\section{Consent for publication}

All authors have read and agreed with the submission of the manuscript. This manuscript has not been published or presented elsewhere in part or in entirety. There are no conflicts of interest to declare.

\section{Competing interests}

All authors declare no conflict of interest.

\section{Author details}

'Department of Cardiovascular Surgery, School of Medicine, The First Affiliated Hospital of Zhejiang University, Number 79 Qingchun Road, Hangzhou, China.
${ }^{2}$ Department of Cardiology, School of Medicine, The First Affiliated Hospital of Zhejiang University, Hangzhou, China.

Received: 7 May 2021 Accepted: 5 July 2021

Published online: 15 July 2021

\section{References}

1. Taylor CJ, Ordonez-Mena JM, Roalfe AK, Lay-Flurrie S, Jones NR, Marshall T, Hobbs FDR. Trends in survival after a diagnosis of heart failure in the United Kingdom 2000-2017: population based cohort study. BMJ. 2019:364:1223.

2. Frey N, Olson EN. Cardiac hypertrophy: the good, the bad, and the ugly. Annu Rev Physiol. 2003;65:45-79.

3. Harvey PA, Leinwand LA. The cell biology of disease: cellular mechanisms of cardiomyopathy. J Cell Biol. 2011;194(3):355-65.

4. Tariq U, Uppulapu SK, Banerjee SK. Role of GSK-3 in Cardiac Health: Focusing on Cardiac Remodeling and Heart Failure. Curr Drug Targets 2021

5. Zhao Y, Chen Y, Jin M, Wang J. The crosstalk between m(6)A RNA methylation and other epigenetic regulators: a novel perspective in epigenetic remodeling. Theranostics. 2021;11(9):4549-66.

6. Tang L, Wei X, Li T, Chen Y, Dai Z, Lu C, Zheng G. Emerging perspectives of RNA N (6)-methyladenosine (m(6)A) modification on immunity and autoimmune diseases. Front Immunol. 2021;12:630358.

7. Jiang X, Liu B, Nie Z, Duan L, Xiong Q, Jin Z, Yang C, Chen Y. The role of m6A modification in the biological functions and diseases. Signal Transduct Target Ther. 2021;6(1):74.

8. Li X, Yang Y, Chen S, Zhou J, Li J, Cheng Y. Epigenetics-based therapeutics for myocardial fibrosis. Life Sci. 2021:271:119186.

9. Kim J, Lee G. Metabolic Control of m(6)A RNA Modification. Metabolites. 2021;11(2):80.

10. Gu J, Zhan Y, Zhuo L, Zhang Q, Li G, Li Q, Qi S, Zhu J, Lv Q, Shen Y, Guo Y, Liu S, Xie T, Sui X. Biological functions of m(6)A methyltransferases. Cell Biosci. 2021;11(1):15.

11. Fang X, Li M, Yu T, Liu G, Wang J. Reversible N6-methyladenosine of RNA: The regulatory mechanisms on gene expression and implications in physiology and pathology. Genes Dis. 2020;7(4):585-97.

12. Wang $X$, Zhao BS, Roundtree IA, Lu Z, Han D, Ma H, Weng X, Chen K, Shi $\mathrm{H}, \mathrm{He} \mathrm{C}$. N(6)-methyladenosine modulates messenger RNA translation efficiency. Cell. 2015;161(6):1388-99.

13. Du H, Zhao Y, He J, Zhang Y, Xi H, Liu M, Ma J, Wu L. YTHDF2 destabilizes m(6)A-containing RNA through direct recruitment of the CCR4NOT deadenylase complex. Nat Commun. 2016;7:12626.

14. Liu XM, Zhou J. Multifaceted regulation of translation by the epitranscriptomic modification N(6)-methyladenosine. Crit Rev Biochem Mol Biol. 2021;56(2):137-48.

15. Berulava T, Buchholz E, Elerdashvili V, Pena T, Islam MR, Lbik D, Mohamed BA, Renner A, von Lewinski D, Sacherer M, Bohnsack KE, Bohnsack MT, Jain G, Capece V, Cleve N, Burkhardt S, Hasenfuss G, Fischer A, Toischer K. Changes in m6A RNA methylation contribute to heart failure progression by modulating translation. Eur J Heart Fail. 2020;22(1):54-66.

16. Dorn LE, Lasman L, Chen J, Xu X, Hund TJ, Medvedovic M, Hanna JH, van Berlo JH, Accornero F. The N(6)-Methyladenosine mRNA Methylase METTL3 controls cardiac homeostasis and hypertrophy. Circulation. 2019;139(4):533-45.

17. Ren Z, Yu P, Li D, Li Z, Liao Y, Wang Y, Zhou B, Wang L. Single-cell reconstruction of progression trajectory reveals intervention principles in pathological cardiac hypertrophy. Circulation. 2020;141(21):1704-19.

18. Roxana ME, Georgica T, lonut D, Gianina M, Cristina F. Atrial and brain natriuretic peptides- benefits and limits of their use in cardiovascular diseases. Curr Cardiol Rev. 2019;15(4):283-90.

19. Magri D, Mastromarino V, Gallo G, Zachara E, Re F, Agostoni P, Giordano D, Rubattu S, Forte M, Cotugno M, Torrisi MR, Petrucci S, Germani A, Savio C, Maruotti A, Volpe M, Autore C, Piane M, Musumeci B. Risk stratification in hypertrophic cardiomyopathy insights from genetic analysis and cardiopulmonary exercise testing. J Clin Med. 2020;9(6):1636. 
20. Li Y, Bedi RK, Moroz-Omori EV, Caflisch A. Structural and Dynamic Insights into Redundant Function of YTHDF Proteins. J Chem Inf Model. 2020;60(12):5932-5.

21. Shi R, Ying S, Li Y, Zhu L, Wang X, Jin H. Linking the YTH domain to cancer: the importance of YTH family proteins in epigenetics. Cell Death Dis. 2021;12(4):346.

22. Chen H, Pugh BF. What do Transcription Factors Interact With? J Mol Biol 2021:166883.

23. Ruthenburg AJ, Allis CD, Wysocka J. Methylation of lysine 4 on histone H3: intricacy of writing and reading a single epigenetic mark. Mol Cell. 2007;25(1):15-30.

24. Stein AB, Jones TA, Herron TJ, Patel SR, Day SM, Noujaim SF, Milstein ML, Klos M, Furspan PB, Jalife J, Dressler GR. Loss of H3K4 methylation destabilizes gene expression patterns and physiological functions in adult murine cardiomyocytes. J Clin Invest. 2011;121(7):2641-50.

25. Panda AC, Martindale JL, Gorospe M. Polysome Fractionation to Analyze mRNA Distribution Profiles. Bio Protoc 2017;7(3).

26. Schwanhäusser B, Busse D, Li N, Dittmar G, Schuchhardt J, Wolf J, Chen W, Selbach M. Global quantification of mammalian gene expression control. Nature. 2011:473(7347):337-42.

27. Berezin A. Epigenetics in heart failure phenotypes. BBA Clin. 2016;6:31-7.

28. Hinger SA, Wei J, Dorn LE, Whitson BA, Janssen PML, He C, Accornero F. Remodeling of the $\mathrm{m}(6) \mathrm{A}$ landscape in the heart reveals few conserved post-transcriptional events underlying cardiomyocyte hypertrophy. J Mol Cell Cardiol. 2021;151:46-55.

29. Mathiyalagan $P$, Adamiak M, Mayourian J, Sassi Y, Liang Y, Agarwal N, Jha D, Zhang S, Kohlbrenner E, Chepurko E, Chen J, Trivieri MG, Singh R, Bouchareb R, Fish K, Ishikawa K, Lebeche D, Hajjar RJ, Sahoo S. FTODependent N(6)-Methyladenosine Regulates Cardiac Function During Remodeling and Repair. Circulation. 2019;139(4):518-32.

30. Wang X, Lu Z, Gomez A, Hon GC, Yue Y, Han D, Fu Y, Parisien M, Dai Q, Jia G, Ren B, Pan T, He C. N6-methyladenosine-dependent regulation of messenger RNA stability. Nature. 2014;505(7481):117-20.

31. Shi H, Wang X, Lu Z, Zhao BS, Ma H, Hsu PJ, Liu C, He C. YTHDF3 facilitates translation and decay of N(6)-methyladenosine-modified RNA. Cell Res. 2017:27(3):315-28.

32. Colegrave M, Peckham M. Structural implications of beta-cardiac myosin heavy chain mutations in human disease. Anat Rec (Hoboken). 2014;297(9):1670-80.

33. Lee SP, Ashley EA, Homburger J, Caleshu C, Green EM, Jacoby D, Colan SD, Arteaga-Fernandez E, Day SM, Girolami F, Olivotto I, Michels M, Ho CY, Perez MV, Investigators SH. Incident atrial fibrillation is associated With MYH7 sarcomeric gene variation in hypertrophic cardiomyopathy. Circ Heart Fail. 2018:11(9):005191.

34. Wu R, Yao Y, Jiang Q, Cai M, Liu Q, Wang Y, Wang X. Epigallocatechin gallate targets FTO and inhibits adipogenesis in an mRNA m(6)A-YTHDF2dependent manner. Int J Obes (Lond). 2018;42(7):1378-88.

35. Xie H, Li J, Ying Y, Yan H, Jin K, Ma X, He L, Xu X, Liu B, Wang X, Zheng $X$, Xie L. METTL3/YTHDF2 m(6) A axis promotes tumorigenesis by degrading SETD7 and KLF4 mRNAs in bladder cancer. J Cell Mol Med. 2020;24(7):4092-104.

36. Yu R, Li Q, Feng Z, Cai L, Xu Q. m6A Reader YTHDF2 regulates LPS-induced inflammatory response. Int J Mol Sci. 2019;20(6):1323.

37. Walsh-Wilkinson E, Beaumont C, Drolet MC, Roy EM, Le Houillier C, Beaudoin J, Arsenault M, Couet J. Effects of the loss of estrogen on the heart's hypertrophic response to chronic left ventricle volume overload in rats. PeerJ. 2019;7:7924.

38. Bury L, Megy K, Stephens JC, Grassi L, Greene D, Gleadall N, Althaus K, Allsup D, Bariana TK, Bonduel M, Butta NV, Collins P, Curry N, Deevi SV, Downes K, Duarte D, Elliott K, Falcinelli E, Furie B, Keeling D, Lambert MP, Linger R, Mangles S, Mapeta R, Millar CM, Penkett C, Perry DJ, Stirrups KE, Turro E, Westbury SK, Wu J, BioResource N, Gomez K, Freson K, Ouwehand WH, Gresele P, Simeoni I. Next-generation sequencing for the diagnosis of MYH9-RD: Predicting pathogenic variants. Hum Mutat. 2020;41(1):277-90

39. Chen $M$, Yi B, Sun J. Inhibition of cardiomyocyte hypertrophy by protein arginine methyltransferase 5. J Biol Chem. 2014;289(35):24325-35.

40. Lompre AM, Schwartz K, d'Albis A, Lacombe G, Van Thiem N, Swynghedauw $B$. Myosin isoenzyme redistribution in chronic heart overload. Nature. 1979;282(5734):105-7.

41. Mercadier JJ, Lompré AM, Wisnewsky C, Samuel JL, Bercovici J, Swynghedauw B, Schwartz K. Myosin isoenzyme changes in several models of rat cardiac hypertrophy. Circ Res. 1981;49(2):525-32.

42. Peng HB, Wolosewick JJ, Cheng PC. The development of myofibrils in cultured muscle cells: a whole-mount and thin-section electron microscopic study. Dev Biol. 1981;88(1):121-36.

43. Schiaffino S, Gorza L, Saggin L, Valfré C, Sartore S. Myosin changes in hypertrophied human atrial and ventricular myocardium A correlated immunofluorescence and quantitative immunochemical study on serial cryosections. Eur Heart J. 1984;5(Suppl F):95-102.

44. Bouvagnet $\mathrm{P}$, Mairhofer $\mathrm{H}$, Leger JO, Puech $\mathrm{P}$, Leger JJ. Distribution pattern of alpha and beta myosin in normal and diseased human ventricular myocardium. Basic Res Cardiol. 1989;84(1):91-102.

45. Tsuchimochi H, Sugi M, Kuroo M, Ueda S, Takaku F, Furuta S, Shirai T, Yazaki Y. Isozymic changes in myosin of human atrial myocardium induced by overload Immunohistochemical study using monoclonal antibodies. J Clin Invest. 1984;74(2):662-5.

46. Wang J, Guo X, Dhalla NS. Modification of myosin protein and gene expression in failing hearts due to myocardial infarction by enalapril or losartan. Biochim Biophys Acta. 2004;1690(2):177-84.

47. Nakao K, Minobe W, Roden R, Bristow MR, Leinwand LA. Myosin heavy chain gene expression in human heart failure. J Clin Invest. 1997; 100(9):2362-70.

48. Lowes BD, Minobe W, Abraham WT, Rizeq MN, Bohlmeyer TJ, Quaife RA, Roden RL, Dutcher DL, Robertson AD, Voelkel NF, Badesch DB, Groves BM, Gilbert EM, Bristow MR. Changes in gene expression in the intact human heart Downregulation of alpha-myosin heavy chain in hypertrophied, failing ventricular myocardium. J Clin Invest. 1997;100(9):2315-24.

49. Krenz M, Sanbe A, Bouyer-Dalloz F, Gulick J, Klevitsky R, Hewett TE, Osinska HE, Lorenz JN, Brosseau C, Federico A, Alpert NR, Warshaw DM, Perryman MB, Helmke SM, Robbins J. Analysis of myosin heavy chain functionality in the heart. J Biol Chem. 2003;278(19):17466-74.

50. Goldenberg JR, Carley AN, Ji R, Zhang X, Fasano M, Schulze PC, Lewandowski ED. Preservation of Acyl Coenzyme A Attenuates Pathological and Metabolic Cardiac Remodeling Through Selective Lipid Trafficking. Circulation. 2019;139(24):2765-77.

\section{Publisher's Note}

Springer Nature remains neutral with regard to jurisdictional claims in published maps and institutional affiliations. 